Original Research

\title{
Quantitative Ultrasound of Kidneys, Liver, and Spleen: a Comparison Between Mules and Horses
}

\author{
Maria Cristina Reis Castiglioni, Michel de Campos Vettorato, Jéssica Leite Fogaça, \\ José Nicolau Próspero Puoli Filho, Vânia Maria de Vasconcelos Machado*
}

São Paulo State University (Unesp), School of Veterinary Medicine and Animal Science, Botucatu, Brazil

\section{A R T I C L E I N F O}

\section{Article history:}

Received 4 April 2018

Received in revised form

5 July 2018

Accepted 14 July 2018

Available online 20 August 2018

\section{Keywords:}

Gray-level histogram

Equine

Kidney

Ultrasonographic features

\begin{abstract}
A B S T R A C T
An ultrasonographic evaluation of the renal morphology is subjective in veterinary science. The echogenicity and echotexture are compared among the cortex, medulla, liver, and spleen. The purpose of this study was to quantify the renal echogenicity and echotexture of healthy adult horses and mules. An ultrasonographic examination was performed on 12 horses and 12 mules to obtain bidimensional images of the renal cortex, renal medulla, liver, and spleen. These images were analyzed using a gray-level histogram to obtain the quantitative values of echogenicity, echotexture, and the proportions of each region. Difference between the kidneys of mules and horses was observed. The renal cortex was less echoic ( $6.59 \pm 1.69$ less in mules; $3.04 \pm 1.03$ less in horses $)$ and more homogeneous $(0.17 \pm 0.24$ more in mules; $0.47 \pm 0.22$ more in horses) than the spleen but more hypoechoic ( $1.46 \pm 0.37$ more in mules; 1.05 \pm 1.07 more in horses) and homogeneous ( $0.81 \pm 0.11$ more in mules; $0.85 \pm 0.26$ more in horses) than the liver. In comparing the renal cortex to the medulla, it was observed that the renal cortex is more echoic (up to $3.49 \pm 1.59$ more in mules; $2.07 \pm 0.21$ more in horses) and heterogeneous (up to $0.45 \pm$ 0.23 more in mules; $0.63 \pm 0.09$ more in horses). The most important difference between mules and horses was a more hypoechoic and homogeneous renal medulla in mules, indicating a higher water content of the renal medulla in mules than in horses.
\end{abstract}

(C) 2018 Elsevier Inc. All rights reserved.

\section{Introduction}

Renal ultrasonographic examination is a simple, noninvasive, and fast method that can be performed in nonsedated standing horses $[1,2]$. Standard B-mode permits the morphological characterization of tissues and the detection of changes [3-5]. Ultrasonographic characteristics are shaped by intrinsic (tissue integrity, body condition, hydration level, and gastrointestinal motility) and

\footnotetext{
Animal welfare/ethical statement: The protocol n 08/2016-CEUA, regarding the study "Renal resistive index characterization and renal ultrasonographic and dopplefluxometric padronization of mules” from Maria Cristina Reis Castiglioni, was approved by the Ethical Committee for the Use of Animals in Research (CEUA) of the School of Veterinary Medicine and Animal Science of the São Paulo State University - UNESP on February 12th, 2016.

Conflict of interest statement: The authors declare no conflicts of interest.

* Corresponding author at: Vânia Maria de Vasconcelos Machado, Departamento de Reprodução Animal e Radiologia Veterinária, Faculdade de Medicina Veterinária e Zootecnia, Rua Prof. Dr. Walter Maurício Correa s/n ${ }^{\circ}$ 18.618-681, Botucatu, SP, Brazil.

E-mail address: vania.mv.machado@unesp.br (V.M.V. Machado).
}

extrinsic (ultrasonographer's experience and knowledge, as well as ultrasound settings including time gain compensation, frequency, and focal position) factors [6-11]. An evaluation of the renal characteristics is performed comparing the renal zones between each other and between others tissues, such as the liver and spleen $[1,2,8]$. Some of these variables are subjective and are susceptible to variations and errors, making it necessary to use complementary techniques to increase the accuracy of the examination.

The gray-level histogram (GLH) is an accessible, easy, and efficient method to evaluate ultrasonographic images, allowing the objective characterization of the tissue's echogenicity and echotexture $[6,7,12]$. The GLH consists of the analysis and rating of grays tones in a region of interest, providing the distribution, descriptive analysis, and objective values of echogenicity and echotexture $[6,7,12]$. This complementary technique is underused in veterinary science and there is a lack of studies about this technique in horses. This study aims to quantify the echogenicity and echotexture of the kidneys, liver, and spleen in mules and horses, to obtain the ratios between those regions for comparison, and to provide reference values for a more objective evaluation of equine renal parenchyma. 


\section{Materials and Methods}

\subsection{Sample Population}

Twenty-four equines were used for this study: 12 crossbred horses and 12 mules of similar weight, body condition score, and age. Only healthy animals, based on clinical examination, blood count, serum biochemistry, and urinalysis were selected. This study is in accordance with the ethical principles adopted by the National Council of Animal Control and Experimentation and by the Ethical Commission in Animal Use of this faculty.

\subsection{Images Acquisition}

The ultrasound exam was performed in an open, covered, and well-ventilated environment, using mobile ultrasound equipment (MyLab 30; Esaote, Genoa, Italy) and a multifrequency convex transducer (3.0-9.0 MHz). Fasting was not performed, and chemical restraint was not required. The screening windows of each kidney, the head of the spleen, and the right dorsocaudal region of the hepatic parenchyma were located by ultrasound, clipped, and soaked with $70 \%$ isopropyl alcohol and acoustic gel.

Using longitudinal and transverse imaging planes, the images were obtained using constant values of frequency and gain (3.5 MHz and 61\%) (Fig. 1). All sonographic images were posteriorly saved as still images in the JPEG format.

\subsection{Gray-Level Histogram}

All sonographic images were evaluated by the author (M.C.) and one of the coauthors (M.V.). Only images with adequate definition of the parenchyma and cortical-medullar junction were used for GLH. For the renal cortex and medulla evaluation, a square ROI of $0.5 \mathrm{~cm}^{2}$ (500-1000 pixels) was selected in each region, avoiding extremities or other renal structures, such as the renal capsule, the cortical-medullar junction, diverticula, or vessels (Fig. 2). For the liver and spleen, the ROIs had $1.0 \mathrm{~cm}^{2}$ (1500-2000 pixels) (Fig. 2).

A GLH analysis was performed using ImageJ software version $1.51 \mathrm{k}$ (National Institutes of Health, Bethesda, USA), with a gray scale of 255 shades. The following quantitative parameters were obtained from each ROI: the number of pixels (COUNT), the most frequent gray tonality (MODE), the number of pixels with the MODE value (MODE COUNT), and the pixel's mean (MEAN), standard deviation and minimum and maximum values (Fig. 16). The MEAN and MODE variables were used to analyze the ROI's echogenicity, and a MEAN ratio was created to compare the echogenicity between the liver ( $\mathrm{Li}$ ) and the right kidney cortex (RKC), the RKC and the right kidney medulla (RKM), the left kidney cortex (LKC) and the left kidney medulla (LKM), and the spleen (Sp) and the LKC. The ratio between MODE COUNT and COUNT (MC:C ratio) of each ROI was calculated to evaluate the echotexture. Posteriorly, the MC: $C$ ratio between the ROIs was also compared.

\subsection{Statistical Analysis}

Statistical analysis was performed using the GraphPad Prism program v. 7.00 for Windows (GraphPad Software, San Diego, CA) with statistical significance set at $P<.05$. The MEAN, MODE, MC:C ratio, and MEAN ratio were submitted to the Shapiro-Wilk normality test and descriptive analysis. To observe the difference between the organs (kidney, liver, and spleen), renal zones (cortical and medulla), and animals (horse and mules), an unpaired test was used for variables with normal distribution and the Mann-Whitney test was used for those without.

\section{Results}

A total of 48 renal cortex, 48 renal medulla, 24 hepatic, and 24 splenic regions were analyzed by GLH, and at least 2 values of each ROI were obtained. The descriptive values of MEAN, MODE, MC:C ratio, and MEAN ratio can be found in Tables 1 and 2 . There was no difference observed between the MEAN and MODE values of each ROI $(P<.05)$, but a difference was noticed between regions $(P<$ $.05)$. Comparing horses to mules, a similarity of MEAN and MODE in the hepatic parenchyma was observed, with a difference between other ROIs. When comparing the MC:C and MEAN ratios between horses and mules, it was possible to observe a difference between the RKC:RKM, LKC:LKM, and Sp:LKC ratios but not the Li:RKC ratio.

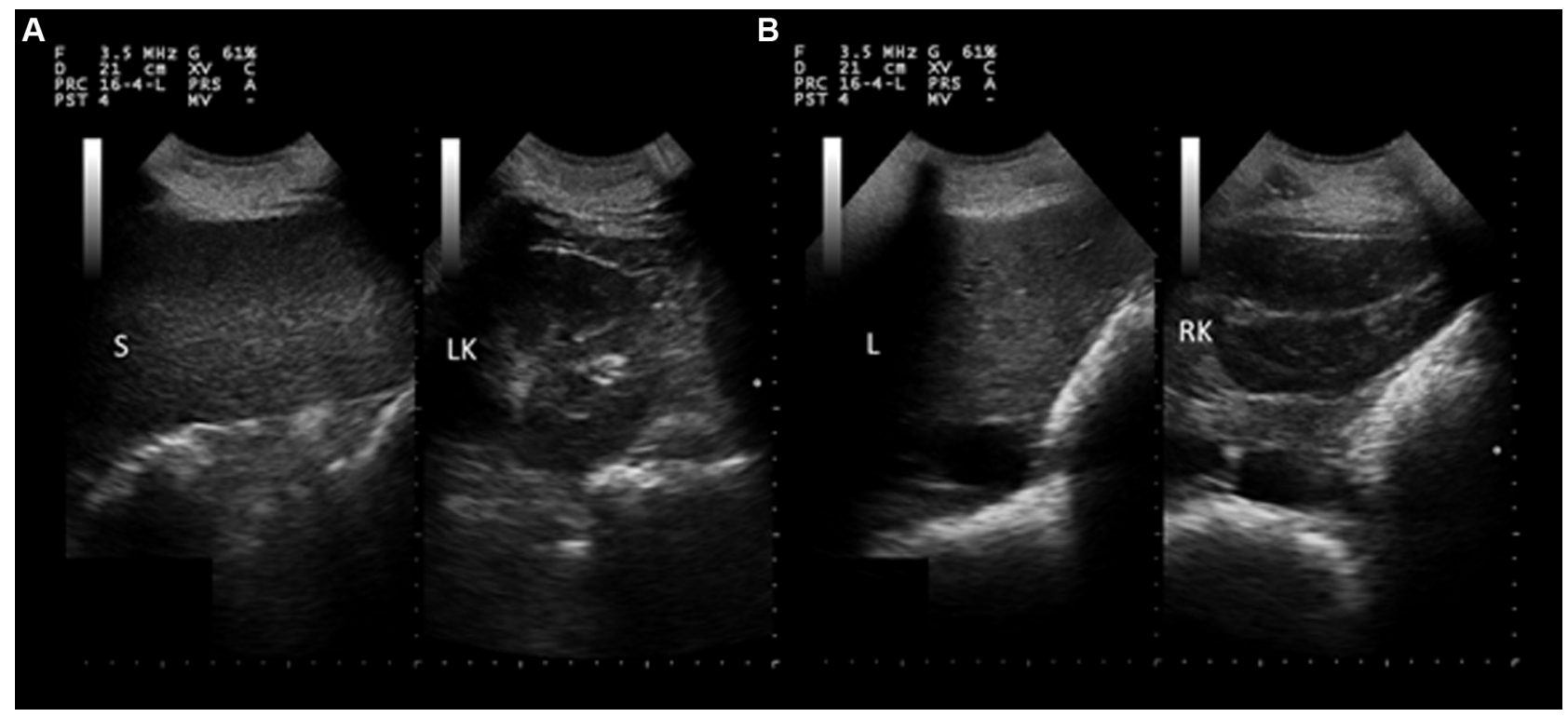

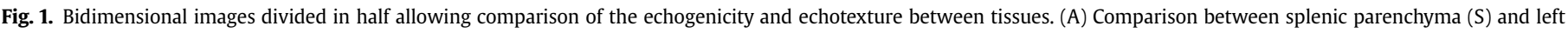
kidney (LK). (B) Comparison between hepatic parenchyma (L) and right kidney (RK). 

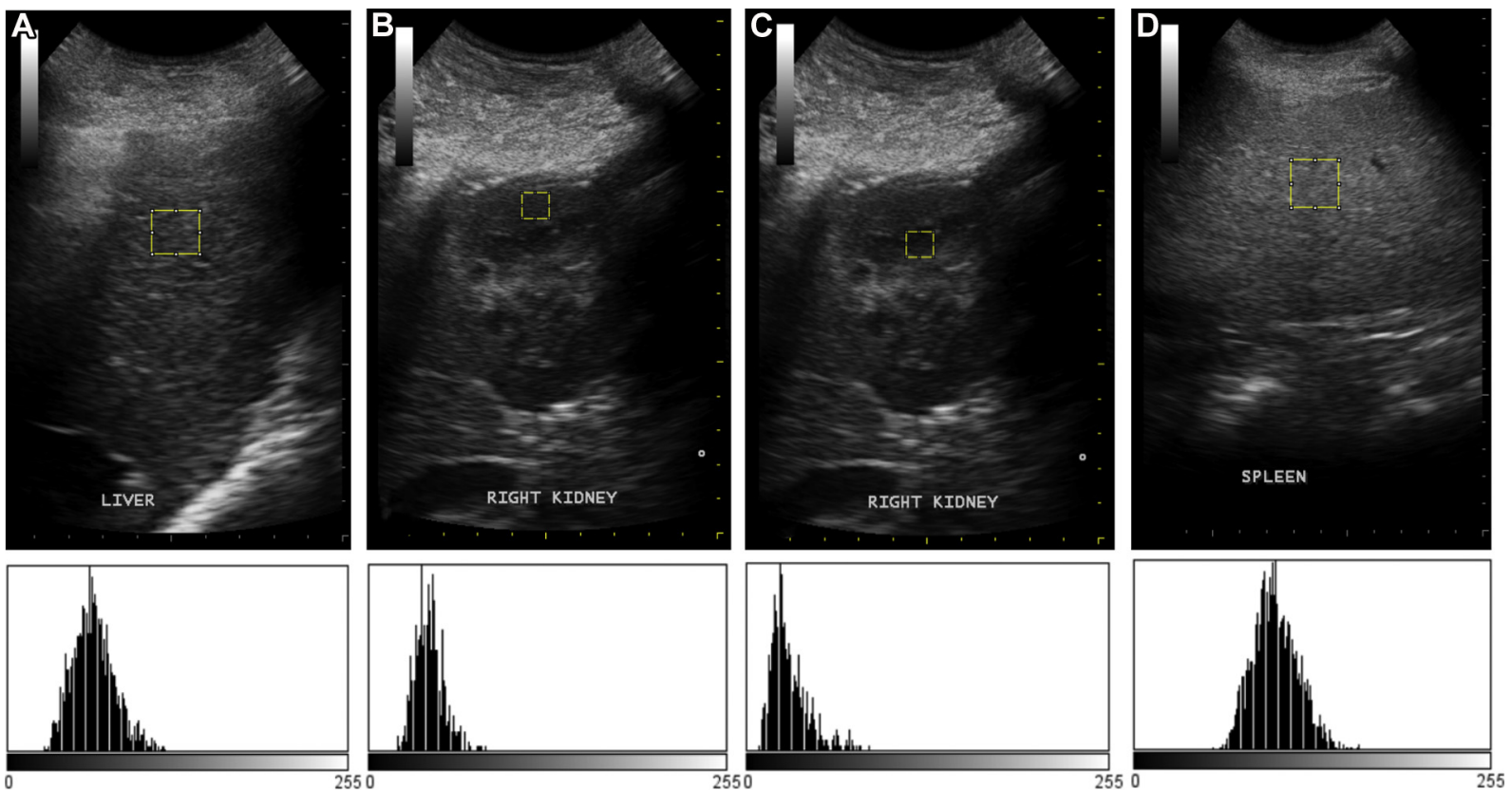

Count 1760

Mean: 64.123

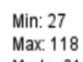

Mode: 61 (66)

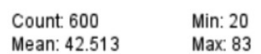

StdDev: 10.115

Count 575

StdDev: 13.624

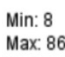

Mode: $23(35)$

Count: 1936

Mean: 100.995 StdDev 16.303
Min: 56

Mode 101 (65)

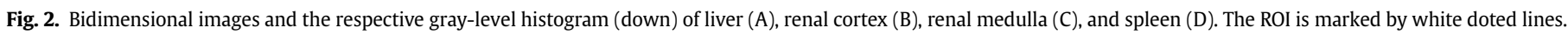
$\mathrm{ROI}$, region of interest.

In mules, the renal cortex is 3 times more echoic than the medulla, approximately 6 times less than the Sp and 1.5 times less than the Li. In mares, the renal cortex is approximately 2 times more echoic than the medulla, 3 times less than the Sp, and almost similar to the $\mathrm{Li}$ (ratio of 1.05). It is possible to observe an MC:C ratio of 0.81 between the Li:RKC ratio in mules, suggesting that the hepatic parenchyma is more heterogeneous. The same is observed between Sp:LKC due to a ratio of 0.17 , but when comparing RKC:RKM and LKC:LKM, the ratios are 0.45 and 0.28 , indicating that the medulla is more homogeneous in both kidneys. The same types of results are observed in mares, except with higher ratio values, indicating higher differences between ROIs.

\section{Discussion}

Even though diagnostic images can be generated in high resolution scales, of 4096-65536 grays tones, they are still evaluated in medical systems with lower resolution scales of 256-1024 gray tones, hindering the detection of subtle or initial lesions and compromising the diagnostic evaluation [13]. In addition, this interference is insignificant because humans have a limited capacity to differentiate between grays tones, being capable of distinguishing only 600-1000 gray tones, according to the environment luminosity conditions [13-15]. In addition, the tissue echogenicity and echotexture are the result of the interaction between the propagation velocity of the sound wave and the acoustic properties of the tissues; therefore, according to the acoustic properties, the interaction can result in different degrees of sound propagation, absorption, reflection, and diffraction, defining the characteristics of the returning echo and, thus, the location and intensity of the gray tone in the ultrasound image $[15,16]$. Therefore, sonographic echogenicity and echotexture evaluation are considered subjective and unsafe methods to detect or control discrete changes [1,6-11,15].

Quantitative analysis provides more accurate values of the tissue characteristics, such as echogenicity and echotexture, and can be performed during or following the sonographic examination $[6,7,12]$. In GLH, the variables MEAN and MODE permit an echogenicity analysis with higher values to infer a more echoic to hyperechoic region and lower values to infer an echoic to hypoechoic region. Differences between the values of MEAN and MODE indicate a nonparametric distribution of the grayscale that occurs due to high contrast, inadequate sample size, or localization. In this study, there was no difference between these variables to infer a high variability of gray tones in the ROI and inadequate sampling. The arrangement of gray tones of an image determinates its echotexture. In GLH, the ratio between the MODE COUNT and the COUNT (MC:C ratio) permits its analysis. The division of the number of MODE pixels (MODE COUNT) by the total number of pixels in an ROI (COUNT), with posterior multiplication by $100 \%$, allows for the evaluation of how much the MODE (the most common tone) represents the ROI. In this case, higher ratios infer that the MODE is representative of the ROI, and thus a more homogeneous echotexture. Lower values indicate the opposite and are less representative and more heterogeneous.

The findings of this study partially coincide with those described by the literature: the renal cortex is more hypoechoic and homogeneous than the hepatic or splenic parenchyma but more echoic and heterogeneous than the renal medulla $[1,2,10,17]$. However, while it is noted that $\mathrm{Sp}$ is more homogeneous than $\mathrm{Li}$, the opposite was observed $[1,2,8]$.

Lower values of MEAN and MODE were found in mules, even though the same sonographic parameters were used, and this difference could be associated with the subcutaneous adipose tissue, which lowers the tissue echogenicity due to increasing sound beam attenuation [16]. Members of the Equus genus have similar sites of subcutaneous adipose tissue, but it is possible to observe the interspecies and intraspecies variations in the sites with priority of deposition and mobilization due to metabolic and physiologic differences $[18,19]$. Donkeys are different from domestic horses, as they have a higher accumulation of subcutaneous adipose tissue in the base of the tail, barrel, neck, and abdominal wall $[18,19]$. 
Table 1

Descriptive analysis of MEAN, MODE, and MODE COUNT:COUNT ratios (\%) of the renal cortex, renal medulla, liver, and spleen from 12 mules and 12 horses.

\begin{tabular}{|c|c|c|c|c|}
\hline ROI & Mean & SD & Median & SE \\
\hline \multicolumn{5}{|l|}{ Horse } \\
\hline \multicolumn{5}{|l|}{ Right Kidney } \\
\hline \multicolumn{5}{|c|}{ Cortex } \\
\hline MEAN & 50.97 & 16.92 & 48.07 & 2.78 \\
\hline MODE & 45.24 & 17.58 & 45.00 & 2.88 \\
\hline rMC:C & 4.85 & 1.38 & 4.74 & 0.23 \\
\hline \multicolumn{5}{|l|}{ Medulla } \\
\hline MEAN & 28.43 & 12.79 & 29.61 & 2.07 \\
\hline MODE & 25.24 & 12.79 & 27.50 & 1.96 \\
\hline rMC:C & 7.88 & 3.46 & 6.98 & 0.56 \\
\hline \multicolumn{5}{|l|}{ Left Kidney } \\
\hline \multicolumn{5}{|l|}{ Cortex } \\
\hline MEAN & 24.41 & 12.46 & 25.31 & 2.23 \\
\hline MODE & 21.48 & 12.61 & 20.00 & 2.26 \\
\hline rMC:C & 9.24 & 7.29 & 1.33 & 1.33 \\
\hline \multicolumn{5}{|l|}{ Medulla } \\
\hline MEAN & 14.29 & 10.44 & 10.95 & 1.87 \\
\hline MODE & 11.52 & 11.52 & 8.00 & 1.82 \\
\hline rMC:C & 15.21 & 10.83 & 12.20 & 1.94 \\
\hline \multicolumn{5}{|l|}{ Spleen } \\
\hline MEAN & 77.61 & 28.78 & 77.73 & 5.64 \\
\hline MODE & 77.73 & 31.31 & 69.50 & 6.14 \\
\hline rMC:C & 3.67 & 0.77 & 3.61 & 0.15 \\
\hline \multicolumn{5}{|l|}{ Liver } \\
\hline MEAN & 54.04 & 23.89 & 48.00 & 5.09 \\
\hline MODE & 48.82 & 24.09 & 42.50 & 5.13 \\
\hline rMC:C & 3.99 & 0.97 & 3.86 & 0.22 \\
\hline \multicolumn{5}{|l|}{ Mule } \\
\hline \multicolumn{5}{|c|}{ Right Kidney } \\
\hline \multicolumn{5}{|l|}{ Cortex } \\
\hline MEAN & 31.17 & 13.58 & 26.58 & 2.47 \\
\hline MODE & 26.73 & 14.90 & 23.50 & 2.72 \\
\hline rMC:C & 6.23 & 1.89 & 5.61 & 0.33 \\
\hline \multicolumn{5}{|l|}{ Medulla } \\
\hline MEAN & 11.05 & 7.74 & 8.87 & 1.41 \\
\hline MODE & 7.30 & 6.75 & 6.50 & 1.23 \\
\hline rMC:C & 17.36 & 16.57 & 11.48 & 3.02 \\
\hline \multicolumn{5}{|l|}{ Left Kidney } \\
\hline \multicolumn{5}{|l|}{ Cortex } \\
\hline MEAN & 7.65 & 7.25 & 6.47 & 1.66 \\
\hline MODE & 4.63 & 7.16 & 2.00 & 1.64 \\
\hline rMC:C & 23.16 & 19.44 & 16.65 & 4.31 \\
\hline \multicolumn{5}{|l|}{ Medulla } \\
\hline MEAN & 2.56 & 3.33 & 1.19 & 0.74 \\
\hline MODE & 1.20 & 2.96 & 0.00 & 0.66 \\
\hline rMC:C & 50.63 & 27.61 & 52.21 & 6.17 \\
\hline \multicolumn{5}{|l|}{ Spleen } \\
\hline MEAN & 45.37 & 27.37 & 32.74 & 6.28 \\
\hline MODE & 38.47 & 27.37 & 28.00 & 6.27 \\
\hline rMC:C & 4.70 & 1.86 & 4.65 & 0.42 \\
\hline \multicolumn{5}{|l|}{ Liver } \\
\hline MEAN & 40.82 & 17.29 & 41.73 & 4.19 \\
\hline MODE & 33.47 & 16.13 & 34.00 & 3.93 \\
\hline rMC:C & 4.45 & 2.07 & 3.71 & 0.50 \\
\hline
\end{tabular}

Abbreviations: ROI, region of interest; rMC:C, ratio Mode Count:Count; SD, standard deviation; SE, standard error.

Shapiro-Wilk normality test.

Because of being a hybrid, mules can possess some of the same sites that donkeys have, which differentiate them from horses and explain the different echogenicity and echotexture between horses and mules. According to Smith and Burden (2013) [19], the body condition evaluation in mules must be a combination of the ones used in horses and donkeys.

Another observed discrepancy was the difference between the kidneys, with the right more echoic and heterogeneous than the left. Even though the 2 structures are histologically similar, different localizations can result in differences between the structures $[15,16,20]$. With only the intercostal musculature and the abdominal wall in the way of the sound wave, the right kidney has a
Table 2

Descriptive analysis of MEAN and MODE COUNT:COUNT ratios from 12 mules and 12 horses.

\begin{tabular}{|c|c|c|c|c|c|c|c|c|}
\hline & Mean & SD & Median & SE & Mean & SD & Median & SE \\
\hline & \multicolumn{4}{|l|}{ MEAN } & \multicolumn{4}{|c|}{ MODE COUNT:COUNT } \\
\hline \multicolumn{9}{|l|}{ Horse } \\
\hline Li:RKC & 1.10 & 0.49 & 1.05 & 0.09 & 0.89 & 0.26 & 0.85 & 0.06 \\
\hline RKC:RKM & 2.15 & 1.05 & 1.95 & 0.17 & 0.68 & 0.35 & 0.61 & 0.05 \\
\hline Sp:LKC & 5.55 & 5.28 & 3.04 & 1.03 & 0.48 & 0.22 & 0.47 & 0.04 \\
\hline LKC:LKM & 2.17 & 1.19 & 2.07 & 0.21 & 0.74 & 0.46 & 0.63 & 0.09 \\
\hline \multicolumn{9}{|l|}{ Mule } \\
\hline Li:RKC & 0.95 & 0.97 & 0.69 & 0.23 & 0.86 & 0.57 & 0.81 & 0.11 \\
\hline RKC:RKM & 4.46 & 3.70 & 3.16 & 0.67 & 0.47 & 0.23 & 0.45 & 0.05 \\
\hline Sp:LKC & 9.34 & 7.38 & 6.59 & 1.69 & 0.27 & 0.24 & 0.17 & 0.24 \\
\hline LKC:LKM & 6.70 & 6.94 & 3.49 & 1.59 & 0.59 & 0.57 & 0.28 & 0.14 \\
\hline
\end{tabular}

Abbreviations: Li, liver; RKC, right kidney cortex; RCM, right kidney medulla; Sp, spleen; LKC, left kidney cortex; LKM, left kidney medulla; SE, standard error; SD, standard deviation.

more superficial location, which is different than the left one that is located deeper due to the presence of the spleen between it and the skin, increasing the sound wave attenuation and reduces renal echogenicity $[2,10]$.

During ultrasonographic examination, this type of interference is normally controlled with gain control and frequency, which modifies the absolute echogenicity of the image and affects the GLH results [20]. In this study, the variables were maintained constant to avoid interference with the GLH analysis, but it resulted in a difference in the echogenicity and echotexture between the kidneys in horses and mules $[15,20]$. Currently, there is not a preexistent value of the gain or frequency to be used for the renal evaluation, probably because each animal, ultrasound equipment, or exam can result in a different interaction between the sound wave and tissue, and there is a high chance of obtaining different gray tones in the same region. These are the most significant limitations of this study and due to this high variability, the MEAN, MODE, and MC:C ratio values become unreliable reference parameters. However, they can still be used in comparisons, allowing for the creation of a comparative method of evaluation that is less subjective than the human eye. Accordingly, it was possible to observe in a more quantitative mode how the tissues correlate according to echogenicity and echotexture.

In this study, the comparison between the MEAN ratios and the ROI confirmed the previous results and allowed a comparison of the echogenicity between the organs of horses and mules. With the exception of the Li:RKC ratio, the other ratios were of higher value in mules, indicating significant differences that could be correlated to the difference of sound wave attenuation or with the tissue's composition between horses and mules. An absence of echogenicity differences between the liver and right renal cortical (Li:RKC ratio) indicates similar MEAN and MODE values between mules and horses, inferring a lack of a significant composition difference. However, the differences between the RKC:RKM and LKC:LKM ratios of the animals indicated differences between the renal medulla echogenicity of mules and horses.

These findings were also observed during the ultrasound examination and it could be inferred that the mules have a more hypoechoic renal medulla than horses, indicating lower tissue density and higher water content [16]. The renal parenchyma in mammals is histologically divided into 2 distinct zones, the cortex and the medulla. The first one is composed of Bowman's capsules, convoluted tubules, an initial portion of collecting ducts, and corresponding vascularization, whereas the second one is represented by Henle loops, the rest of the collecting ducts, and corresponding vascularization [21]. 
This difference between mules and horses could be correlated to the anatomical and physiological characteristics that the mule inherits from its progenitor. Different than horses, donkeys originated from arid and semiarid environments are capable of surviving longs periods without water and abrupt rehydration [21-23]. Donkeys have a thicker renal medulla and longer Henle loops, allowing for a higher concentration of sodium chloride and urea concentration, factors that increase the osmotic pressure, water reabsorption, and urine concentration [21]. Due to this outcome, donkeys and mules have a renal medulla with a higher water content that is more hypoechoic than horses [21].

\section{Conclusion}

In this study, it was observed that in mules, the renal cortex is 6.59 times less echoic and 0.17 times more homogeneous than the spleen (horses: 3.04 more echoic and 0.47 more homogeneous) but is 1.46 times more hypoechoic and 0.81 times more homogeneous than the liver; this outcome is similar to that, which occurs in domestic horses. It was also observed that in mules, the renal cortex is 3.49 more echoic and 0.45 more heterogeneous than the medulla; however, in horses, the renal cortex is 2.07 more echoic and 0.63 more heterogeneous than the medulla, indicating a more hypoechoic and homogeneous renal medulla in mules than in horses, possibly due to a higher water content inherited from donkeys. However, it becomes necessary to undertake further studies on donkeys and animals of different body score conditions to observe if the renal medulla of mules is similar to that of donkeys and if the rations and proportions found in this study can be found with different weights and sizes of mules and horses. In addition, further studies are required to evaluate what changes to the ratios and proportions are seen in animals with clinical disease.

\section{Acknowledgments}

The authors thank S.A. Image, Brazil, for providing the necessary equipment. The primary funder of this project was National Council for Scientific and Technological Development (CNPq), Brazil.

\section{References}

[1] Penninck DG, Eisenberg HM, Teuscher EE, Vrinz A. Equine renal ultrasonography: normal and abnormal. Vet Radiol Ultrasound 1986;27:81-4.
[2] Reef VB. Equine diagnostic ultrasound. 1st ed. Philadelphia: Saunders; 1998.

[3] Geor RJ. Acute renal failure in horses. Vet Clin North Am Equine Pract 2007;23:577-9.

[4] Matoon JS, Nyland TG. Small animal diagnostic ultrasound. 3rd ed. Philadelphia: Elsevier Saunders; 2015.

[5] Penninck D, D’Anjou MC. Atlas of small animal ultrasonography. 2nd ed. Iowa: Wiley Blackwell; 2015.

[6] Ivancić M, Mai W. Qualitative and quantitative comparison of renal vs. hepatic ultrasonographic intensity in healthy dogs. Vet Radiol Ultrasound 2008;49: $368-73$.

[7] Lee CH, Chol JW, Kim KA, et al. Usefulness of standard deviation on the histogram of ultrasound as a quantitative value for hepatic parenchymal echo texture: preliminary study. Ultrasound Med Biol 2006;32:1817-26.

[8] Matthews HK, Toal RL. A review of equine renal imaging techniques. Vet Radiol Ultrasound 1996;37:163-73.

[9] Schott II HC, Woodie JB. Chapter 64: kidneys and ureters. In: Auer JA, Stick JA, editors. Equine surgery. 4th ed. St Louis: Elsevier Saunders; 2012.

[10] Sertich PL, Turner RM. Equine diagnostic ultrasound. 1st ed. Philadelphia: W.B. Saunders Company; 1998

[11] Toribio RE. Essentials of equine renal and urinary tract physiology. Vet Clin North Am Equine Pract 2007;23:533-61.

[12] Maeda K, Utsu M, Yamamoto N, et al. Chapter 4: ultrasonic tissue characterization with gray level histogram width. In: Kurjak A, Arenas JB, editors. Donald school textbook of transvaginal sonograph. 2nd ed. New Delhi: Jaypee Brothers Medical Publisher Ltd; 2013. p. 17-20.

[13] Kimpe T, Tuytschaever T. Increasing the number of gray shades in medical display systems: how much is enough? J Digit Imaging 2007;20:422-32.

[14] Allison JW, Barrm LL, Massoth RJ, et al. Understanding the process of quantitative ultrasonic tissue characterization. Radiographics 1994;14:1099-108. Bifelow TA, Labyed Y. Chapter 4: attenuation compensation and estimation. In: Mamou J, Oelze ML, editors. Quantitative ultrasound in soft tissues. New York: Springer; 2014. p. 71-94.

[15] Smith-Levitin M, Blicstein I, Albrecht-Shach AA, et al. Quantitative assessment of gray-level perception: observers' accuracy is dependent on density differences. Ultrasound Obstet Gynecol 1997;10:346-9.

[16] Ghoshal G, Oelze ML, O’Brien Jr WD. Chapter 2: quantitative ultrasound history and success. In: Mamou J, Oelze ML, editors. Quantitative ultrasound in soft tissues. New York: Springer; 2014. p. 21-42.

[17] Slovis N. Chapter 23: ultrasonography of the liver, spleen, kidney, bladder, and peritoneal cavity. In: Kidd J, Lu KG, Frazer ML, editors. Atlas of equine ultrasonography. West Sussex: John Wiley \& Sons; 2014. p. 409-27.

[18] Quaresma M, Payan-Carreira R, Silva SR. Relationship between ultrasound measurements of body fat reserves and body condition score in female donkeys. Vet J 2013;197:329-34.

[19] Smith DG, Burden FA. Chapter 16: practical donkey and mule nutrition. In: Geor RJ, Harris PA, Coenen M, editors. Equine applied and clinical nutrition: importance of nutrition for health, welfare and performance. London: Elsevier Saunders; 2013. p. 304-18.

[20] Manley JA, O'Neill WC. How echogenic is echogenic? Quantitative acoustics of renal cortex. Am J Kidney Dis 2001;37:706-11.

[21] Hill RW, Wyse GA, Anderson M. Animal physiology. 3rd ed. Massachusetts: Sinauer Associates; 2012.

[22] Kasirer-Izraeli H, Shkolnik A, Choshniak I. Kidney response to rapid rehydration in the donkey. Isr J Zool 2001;47:29-39.

[23] Maloiy GMO. Water economy of the Somali donkey. Am J Physiol 1970;219: $1522-7$. 